\section{A compact differential laser Doppler velocimeter using a semiconductor laser}

\author{
H W Jentink $\uparrow$, J A J van Beurden $\dagger$, M A Helsdingen $\dagger$, \\ F F M de Mul $\dagger$, H E Suichies $\ddagger$, J G Aarnoudse $\ddagger$ and J Greve ${ }^{\dagger}$ \\ $\dagger$ Department of Applied Physics, University of Twente, \\ PO Box 217, 7500 AE Enschede, The Netherlands \\ $\$$ Department of Obstetrics and Gynaecology, University \\ Hospital Groningen, Oostersingel 59, $9713 \mathrm{EZ}$ Groningen, \\ The Netherlands
}

Received 9 June 1986, in final form 3 December 1986

\begin{abstract}
A small differential laser Doppler velocimeter which uses a semiconductor laser and a small number of optical components is described. In this device the light from the laser diode is split into coherent beams by means of a diffraction grating. The two first-order beams are crossed in a probe volume with a lens. In a test experiment the velocity of water containing $0.9 \mu \mathrm{m}$ polystyrene spheres was determined from the fluctuation of the scattered light. This velocity agreed with the velocity calculated from flux measurements.
\end{abstract}

\section{Introduction}

Differential laser Doppler velocimetry is a well known method to determine the velocity of particles in a fluid or a gas. The measurements are performed by crossing two coherent light beams in a small volume. A particle passing through the volume illuminated by both beams will scatter light of both beams in all directions. The frequency of the light scattered by the moving particles is shifted due to the Doppler effect. A difference in frequency shift exists for the light scattered from each beam if the two beams cross each other at a small angle. This difference is for a non-relativistic speed of the particle constant in all directions and has a value (Drain 1980)

$$
\Delta f=\left(2 v_{x} \sin \frac{1}{2} \alpha\right) / \lambda
$$

where $\alpha$ is the angle between the two intersecting beams, $\lambda$ is the wavelength of the light and $v_{x}$ is the velocity component of the particles in the plane of the light beams, perpendicular to the bisector of the two beam directions. This relationship correlates the velocity component to the frequency of the intensity fluctuation on a detector which detects the scattered light. The same relation is found if an analysis is made of the fluctuation of the light intensity due to intersection of the interference pattern of the two beams in a probe volume by the particle (Drain 1980, Lading 1971). This interference pattern consists of planes, perpendicular to the plane of the beams and parallel to the bisector of the beams. The distance between planes with constructive interference is given by

$$
s=\lambda / 2 \sin \frac{1}{2} \alpha .
$$

Usually a HeNe laser or another gas-filled laser is used for the velocity measurements. However, the use of a semiconductor laser (Shaughnessy and Zu'bi 1978, Dubnishchev et al 1984) is very attractive because of its small size and its low costs. To exploit these advantages we devised the optical part of an apparatus in which a semiconductor laser can be used as a light source for differential laser Doppler velocimetry.

\section{The experimental set-up}

A semiconductor laser of the type which is normally used in a compact-disc player or for telecommunication has a small coherence length compared with the coherence length of a gas laser. This means that the two beams formed from the light of the laser must have the same optical pathlength to the scattering region (Wang 1974). Then the beams have a defined phase correlation in the scattering region and interference of light will occur. A good possibility to obtain two coherent light beams is to use a diffraction grating as a beam splitter. This method of beam splitting is also attractive because of the ease of alignment and its small size.

The total set-up is drawn schematically in figure 1. In our set-up a microscope objective (Leitz $32 \times, \mathrm{NA}=0.60$ ) focuses the light $(810 \mathrm{~nm}, 5 \mathrm{~mW})$ from a Matsushita laser diode (type MEL 4745) on the grating which splits the light beam into separate beams, corresponding to directions in which constructive interference occurs. The grating $(50$ lines $/ \mathrm{mm})$ used is a phase grating which diffracts $45 \%$ of the initial intensity into the first-order directions. These first-order light beams are used to form the scattering volume, while the zero, second and higher orders were stopped by a beam stop. The first-order light beams are crossed in the scattering volume by a positive lens (focal length $50 \mathrm{~mm}$ ). With this arrangement a waist-to-waist projection is realised, which means that the waists of the two beams are brought to coincide within the scattering volume.

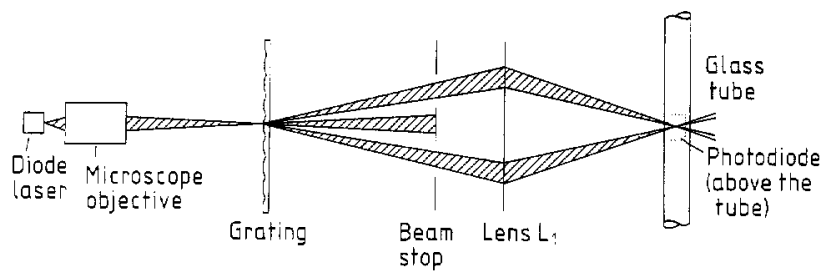

Figure 1. Schematic representation of the laser Doppler velocimeter. The total length of the set-up is $600 \mathrm{~mm}$. The distance between the focusing lens $\mathrm{L}_{1}$ and the middle of the tube is $64 \mathrm{~mm}$. The photodiode can be placed in any scattering direction for the detection of Doppler signals. The cross hatching shows the area of the light beams.

The fluctuating intensity of the scattered light was detected by a Philips (type BPX 40) photodiode. The photodiode signal was analysed by a Hewlett Packard signal analyser after current-voltage conversion. Examples of a measured Doppler burst and a frequency spectrum are shown in figures 3 and 4 .

After demonstrating that the set-up described above worked well, the apparatus was miniaturised. The resulting set-up is shown in figure 2. Instead of the large microscope objective a gradient index lens of length $4.7 \mathrm{~mm}$ and diameter $1.8 \mathrm{~mm}$ was used to focus the light from the Matsushita laser diode on to the

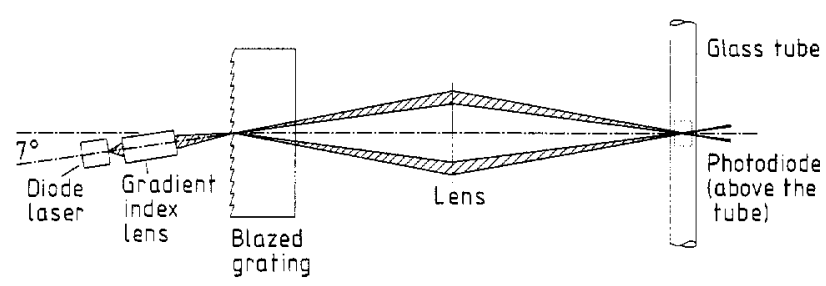

Figure 2. Drawing of the miniaturised laser Doppler velocimeter. The total length of the set-up is $90 \mathrm{~mm}$ 
grating. This grating has 300 lines $/ \mathrm{mm}$ in order to split the two beams to larger angles, which is necessary for miniaturisation. The zero- and first-order diffraction beams of the grating (Bausch \& Lomb type 35-54-04-800) were used to form the probe volume. A lens with $12.5 \mathrm{~mm}$ focus length collimates the beams in the probe volume. The length of the set-up (from laser diode to probe volume) is $90 \mathrm{~mm}$. The other dimensions ( $40 \mathrm{~mm} \times$ $50 \mathrm{~mm}$ ) were determined by the grating we used. These sizes can be reduced to about $20 \mathrm{~mm} \times 20 \mathrm{~mm}$, because only a very small part of the grating is used for the beam splitting.

\section{Results}

The measurements with the miniaturised set-up are comparable with measurements with the first set-up, as will be shown in figure 4. Further results are given for the first set-up.

One of the most important characteristics of a differential laser Doppler system is the size of the probe volume. We minimised the size of the volume by placing the grating in the waist of the light beam formed by the microscope objective. Figure 3 shows the time dependence of the photocurrent when a polystyrene sphere (diameter $0.9 \mu \mathrm{m}$ ) passes through the middle of the probe volume. This probe volume, formed by crossing two beams at an angle $\alpha=10.3^{\circ}$, is located in the middle of a $5 \mathrm{~mm}$ ID glass tube in which water containing $0.9 \mu \mathrm{m}$ polystyrene spheres is circulated. This angle corresponds to a distance between the planes of interference in the probe volume of $3.4 \mu \mathrm{m}$ (in water). Because the photocurrent from the detector is linearly dependent on the light intensity it is possible to calculate the length of the probe volume in the direction perpendicular to the interference planes from this picture. The distance between the points in which there is a factor $1 / \mathrm{e}^{2}$ of the maximum intensity is $60 \mu \mathrm{m}$. In order to be detected a particle has to move through the probe volume defined by the crossing beams. In our set-up this volume is $1.28 \times 10^{-3} \mathrm{~mm}^{3}$.

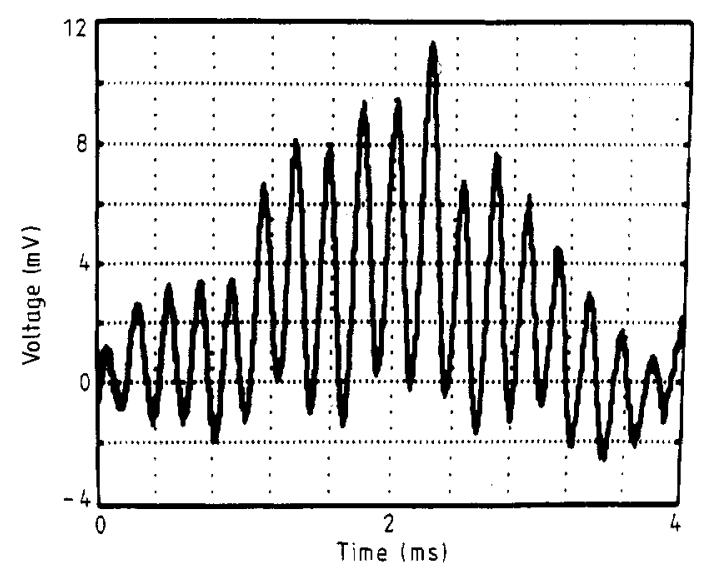

Figure 3. Time dependence of the photocurrent when a particle passes through the probe volume.

In figure 4 spectra of photocurrents are shown with $0.9 \mu \mathrm{m}$ polystyrene spheres passing through the tube. This measurement was made using the set-up described above except for the detection angle, which was altered from forward scatter to $90^{\circ}$ scatter, and the concentration of the spheres, which was increased in order to have a better signal-to-noise ratio. The mean number of particles in the scattering volume when figure 3 was recorded was well below one to reduce the possibility that two or more particles scattered light on the detector at the same time. Figure 5 shows the dependence of the peak frequency on the particle velocity in the centre of the tube. The particle
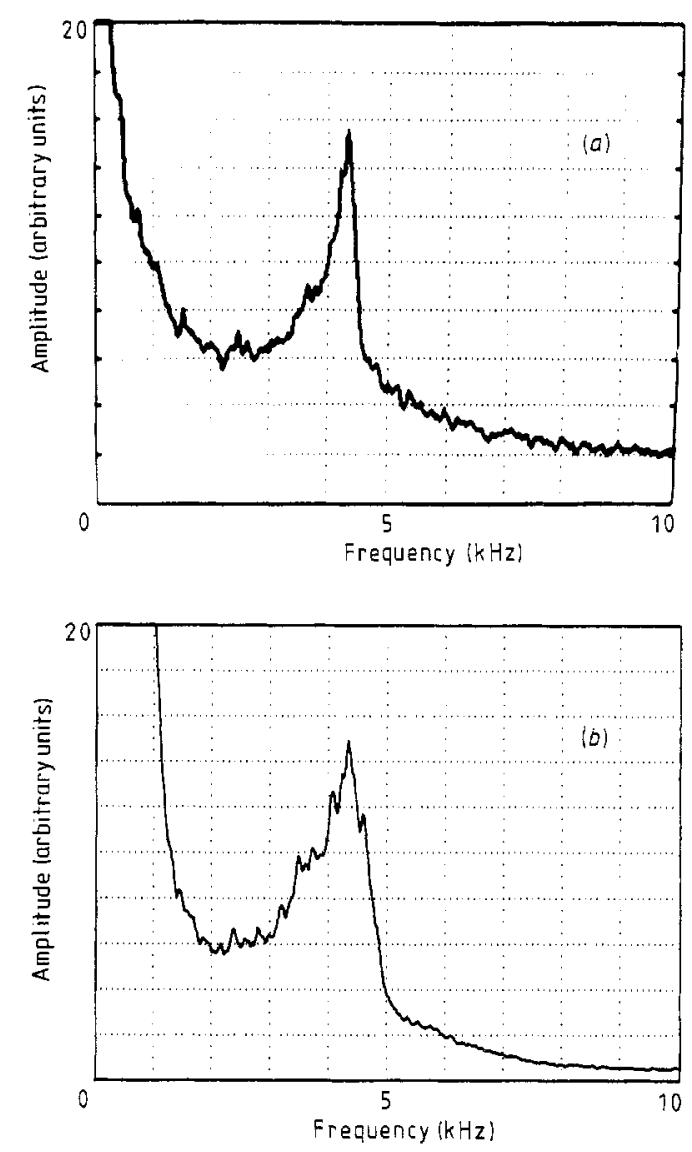

Figure 4. Amplitude spectra of the photocurrents with polystyrene spheres passing the probe volume of the first set-up $(a)$ and the miniaturised set-up (b).

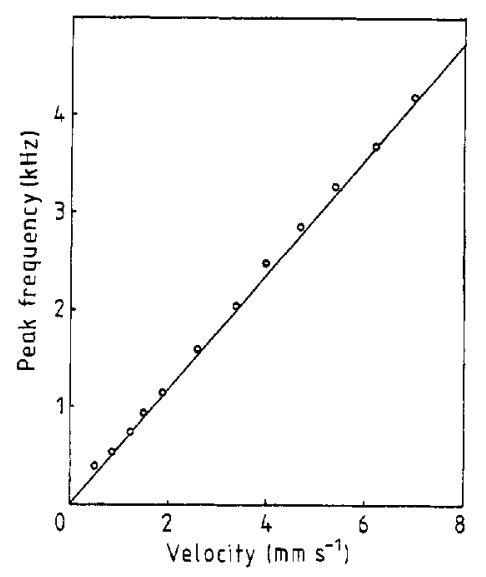

Figure 5. Dependence of the position of the Doppler peak in the frequency spectrum on the particle velocity. The line in this figure shows the expected peak position, which was calculated from equation (1).

velocity is determined by the water flow through the tube. The Reynolds number for the flow

$$
R e=\rho d V / \eta
$$

(where $\rho$ is the fluid density, $d$ is the inner diameter of the tube, $V$ is the mean velocity of the fluid and $\eta$ is the dynamic viscosity) is 20 for the largest velocity. This value is much smaller than 2100 , which means that there is no turbulence (Bird et al 1960). For laminar flow the fluid velocity in the middle of the tube is twice the mean velocity in the tube, which in the experiment was determined from the flux through the tube. 


\section{Conclusions}

With a minimum of optical components a simple and cheap laser Doppler velocimeter can be built. From the results it is clear that the velocity measured by this device agrees well with the real velocity of the particles flowing in the scattering volume.

\section{Acknowledgment}

These investigations are supported by the Netherlands Technology Foundation (STW).

\section{References}

Bird R B, Stewart W E and Lightfoot E N 1960 Transport

Phenomena (New York: Wiley)

Drain L E 1980 The Laser Doppler Technique (Chichester: Wiley)

Dubnishchev Y N, Zhmud V A, Pavlov V A and Stolpovskii A A 1984 Use of a semiconductor laser in a coherent-optics velocimeter

Avtometriya 1 110-1 (transl. Autom. Monit. Meas.)

Lading L 1971 Differential Doppler heterodyning technique Appl. Opt. 10 1943-9

Shaughnessy E J and Zu'bi F H 1978 GaAlAs diode sources for laser-Doppler anemometry

Appl. Phys. Lett. 33 835-6

Wang C P 1974 Doppler velocimeter using diffraction grating

and white light

Appl. Opt. 13 1193-5 